\title{
Association between lower urinary tract symptoms and cigarette smoking or alcohol drinking
}

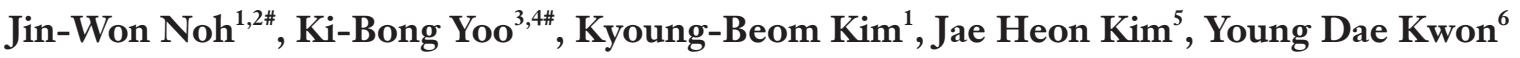 \\ ${ }^{1}$ Department of Health Administration, Dankook University, Cheonan, South Korea; ${ }^{2}$ Global Health Unit, Department of Health Sciences, \\ University Medical Centre Groningen, University of Groningen, Groningen, The Netherlands; ${ }^{3}$ Department of Health Administration, ${ }^{4}$ Department \\ of Information and Statistics, College of Health Sciences, Yonsei University, Wonju, South Korea; ${ }^{5}$ Department of Urology, Soonchunhyang \\ University Hospital, Soonchuhyang University Medical College, Seoul, South Korea; ${ }^{6}$ Department of Humanities and Social Medicine, College of \\ Medicine and Catholic Institute for Healthcare Management, The Catholic University of Korea, Seoul, South Korea \\ Contributions: (I) Conception and design: JW Noh, JH Kim; (II) Administrative support: None; (III) Provision of study materials or patients: None; (IV) \\ Collection and assembly of data: JW Noh, KB Kim; (V) Data analysis and interpretation: JW Noh, KB Kim, YD Kwon; (VI) Manuscript writing: All \\ authors; (VII) Final approval of manuscript: All authors. \\ \#These authors contributed equally to this work. \\ Correspondence to: Jae Heon Kim, MD, PhD. Department of Urology, Soonchunhyang University Seoul Hospital, 59, Daesagwan-ro, Yongsan-gu, \\ Seoul 140-743, South Korea. Email: piacekjh@hanmail.net; piacekjh@schmc.ac.kr.
}

Background: Although there have been several studies about the relationship between alcohol or smoking and lower urinary tracts symptoms (LUTS). This study aimed to investigate the association between alcohol or smoking and severity of LUTS in men, as alcohol intake and cigarette smoking is important modifiable lifestyle factors for LUTS.

Methods: A cross-sectional analysis has been performed and a total of 86,707 participants in Korean Community Health Survey were included for final analysis. The adjusted odds ratio (OR) or coefficient with $95 \%$ confidence interval (CI) estimates were described to show the association between alcohol consumption or cigarette smoking and LUTS.

Results: Among the total subjects, 77,398 (89.3\%), 7,532 (8.7\%), and 1,777 (2.0\%) had mild, moderate, and severe symptoms, respectively, according to International Prostate Symptom Score (IPSS) grade. Those who drank alcohol at least once per month were significantly associated with decreased risk of having the worst IPSS grade (OR: 0.80, 95\% CI: 0.68 to 0.93 ). Those who smoked in the past but currently quitted and those who were daily smokers showed significantly increased risk of having the worst IPSS grade (past smoker, OR: 1.26 , $95 \%$ CI: 1.14 to 1.39 ; daily smoker, OR: 1.21 , $95 \%$ CI: 1.10 to 1.34 ). For nocturia, daily smoking showed positive effect (OR: $0.79,95 \%$ CI: 0.75 to 0.84 ) whereas heavy alcohol drinking showed negative effect (OR: 1.22, 95\% CI: 1.14 to 1.32 )

Conclusions: Alcohol showed positive effect on LUTS except nocturia whereas cigarette smoking had negative effect on LUTS except nocturia. Daily smoking showed positive effect on nocturia whereas heavy alcohol drinking showed negative effect on nocturia.

Keywords: Alcohol; smoking; prostatic hyperplasia; lower urinary tract symptoms

Submitted Oct 30, 2019. Accepted for publication Jan 05, 2020.

doi: $10.21037 /$ tau.2020.03.07

View this article at: http://dx.doi.org/10.21037/tau.2020.03.07 


\section{Introduction}

Traditionally, male lower urinary tract symptoms (LUTS) has been regarded as main problem of benign prostate hyperplasia (BPH) while female LUTS has been regards as overactive bladder $(\mathrm{OAB})$. However, there are emerging evidences showing that not only biological factors of $\mathrm{BPH}$ and $\mathrm{OAB}$, but also modifiable lifestyle factors could affect LUTS $(1,2)$. Constructing changes in these modifiable lifestyle factors are important because there could be a potential treatment to control non-urological origin. However, only a few studies have focused on the efficacy of changing modifiable lifestyle factors including water restriction and weight loss in relation with nocturia and stress urinary incontinence (2-5).

The main reason for few studies on the efficacy of changing modifiable lifestyle factors by prospective trials lies on inconsistent results from many observational studies. Before medication treatment, patients are generally educated to modify their lifestyle factors including changing volume of fluid intake, less caffeine, and less alcohol (1). However, these recommendations could only be adopted by limited portion of patients with LUTS. For better and broader application of changing lifestyle factors, more reliable evidences are needed.

Among different lifestyle factors, cigarette smoking and alcohol drinking have shown inconsistent results regarding their effects on LUTS. Recently, one systematic review study has concluded that modest alcohol intake is a favorable factor for BPH/LUTS (2). However, excessive alcohol intake could lead to worse LUTS compared than less alcohol intake (2). About the relationship between cigarette smoking and LUTS, conclusion could not be made due to too few studies and inconsistent results (2).

Considering the importance of cigarette smoking and alcohol intake for general health of our entire body, more objective studies are needed using large population sample to validate their relationships with LUTS. The objective of the present study was to investigate the relationship of LUTS severity with cigarette smoking and alcohol intake using validated questionnaire.

\section{Methods}

\section{Data and subjects}

This study used data obtained from the 2011 Korean Community Health Survey (KCHS). The KCHS is a nationwide public health survey annually conducted since 2008 by the Korean Centers for Disease Control and Prevention. The purpose of KCHS is to estimate community-based health statistics, morbidity, and prevalence of diseases among Korean adults. The KCHS adopted a multi-stage sampling design to ensure that the sampling unit was representative of the nation's population. In the first stage, a primary sampling unit (district, town, and village) was extracted according to the number of households in each of the smallest governmental administrative units. A probability proportionate to the size sampling method was used when extracting samples. In the second stage, average of five sample households was extracted using systematic sampling methods at selected sampling point and every member of a household who was 19 years or older was interviewed. This study was ultimately carried out using data from 86,707 subjects after excluding 142,519 respondents who were females $(\mathrm{N}=126,209)$, who reported currently receiving treatment with prostatic hyperplasia $(\mathrm{N}=4,745)$ to prevent bias that might affect LUTS, and who had missing data for International Prostate Symptom Score (IPSS), dietary behavior variables, or covariates $(\mathrm{N}=11,565)$ from 229,226 participants originally included in the $2011 \mathrm{KCHS}$ (Figure 1).

\section{Variables and measurements}

This study measured the LUTS of survey participants based on responses from the Korean version of IPSS Questionnaire on KCHS, one of the most widely used tools for evaluating LUTS. As independent variables, frequency of alcohol consumption and smoking status were measured by self-reported questionnaires. Alcohol consumption frequency was classified into "non-drinker", "drink less than once per month", "drink two to four times per month", "drink two to three times per week", and "drink four or more times per week". Smoking status was determined by four categories: daily smoker, non-daily smoker, former smoker (smoked in the past but currently quitted), and never smoker (individuals who had smoked fewer than 100 cigarettes in their lifetime). Dependent variables were IPSS grade \{mild: IPSS total [0-7], moderate [8-19], severe [2035]\}, IPSS voiding [sum of IPSS Q1 (incomplete emptying), Q3 (intermittency), Q5 (weak stream), Q6 (straining); 5 or higher score categorized as having symptoms], IPSS storage [sum of IPSS Q2 (frequency), Q4 (urgency), Q7 (nocturia); 4 or higher score categorized as having symptoms], and nocturia (IPSS Q7; 1 or higher score categorized as having symptoms). 


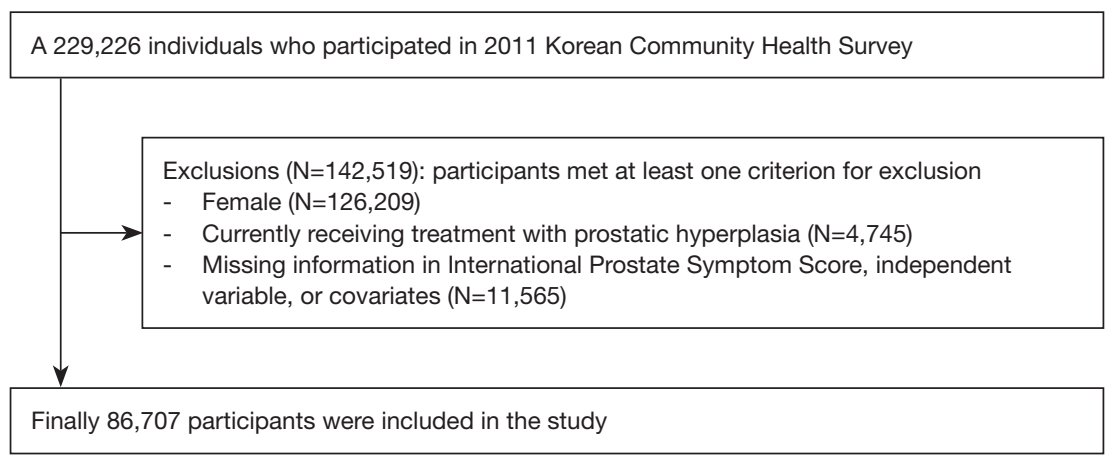

Figure 1 Schematic diagram of the study subject selection.

Socio-demographic factors and comorbidities were considered as covariates. Socio-demographic variables included age, marital status, education level, household income, and residence. Age was divided into "19-29", "30-39", “40-49”, "50-59”, “60-69”, "70-79”, “80-89”, and "90 or higher". Marital status was classified into "married", "separated, divorced, or widowed", and "never married". Education level was classified into "elementary school graduate or lower", "middle school graduate", "high school graduate", and "college graduate or higher". Household income was divided according to quartiles. Residence was based on 16 governmental administrative districts and assigned as "capital" (Seoul), "urban" (6 metropolitan cities were included; Busan, Daegu, Incheon, Gwangju, Daejeon, and Ulsan), or "rural" (rest of capital and urban regions were included: Gyeonggi, Gangwon, Chungbuk, Chungnam, Chungbuk, Chungnam, Jeonbuk, Jeonnam, Gyeongbuk, Gyeongnam, and Jeju). Comorbidities considered hypertension, diabetes mellitus, and dyslipidemia, the most prevalent chronic diseases in Korean adults. They were determined based on physician's diagnosis.

\section{Statistical analysis}

Descriptive analysis was performed to summarize LUTS and socio-demographic characteristics of the study population. The frequency and percentage by IPSS grade were reported. To analyze group differences, a Chi-squared test was conducted. Ordinal logistic regression (for the IPSS grade) and binomial logistic regression (for the IPSS voiding, storage, and nocturia symptoms) were performed to identify the relationship between LUTS measured by IPSS and alcohol consumption or smoking among Korean male adults after controlling for independent variables and covariates. Adjusted odds ratio (OR) with $95 \%$ confidence interval (CI) estimates were reported and threshold for statistical significance was set at $5 \%$ (two-tailed). All analysis applied benchmark weight provided from KCHS to adjust for complex sampling design to produce reliable designbased estimates and ensure national representativeness. Data were analyzed in Stata version 14.2 (StataCorp LP, College Station, TX, USA).

\section{Ethics statement}

Procedures of this study were reviewed and approved by the Institutional Review Board of Soonchunhyang University Seoul Hospital with a waiver for informed consent (201812-011). KCHS data are openly accessible at national public repository (http://chs.cdc.go.kr). There were no confidentiality risks to participants of this study because all KCHS data were deidentified.

\section{Results}

Table 1 summarizes socio-demographic and LUTS characteristics of study subjects. Of a total of 86,707 study subjects, 77,398 (89.3\%), 7,532 (8.7\%), and 1,777 (2.0\%) had mild, moderate, and severe symptoms, respectively, according to IPSS grade. Those who were older, separated, divorced or widowed in marital status, with lower education level or household income, residing in rural region, having hypertension, diabetes mellitus, or dyslipidemia, smoked in past but currently quitted, non-drinker or drinking four or more times per week tended to be have worse condition based on IPSS grade compared to their counterparts. The distribution of study subjects by IPSS grade showed 
Table 1 Characteristics of study participants by International Prostate Symptom Score (IPSS) grade

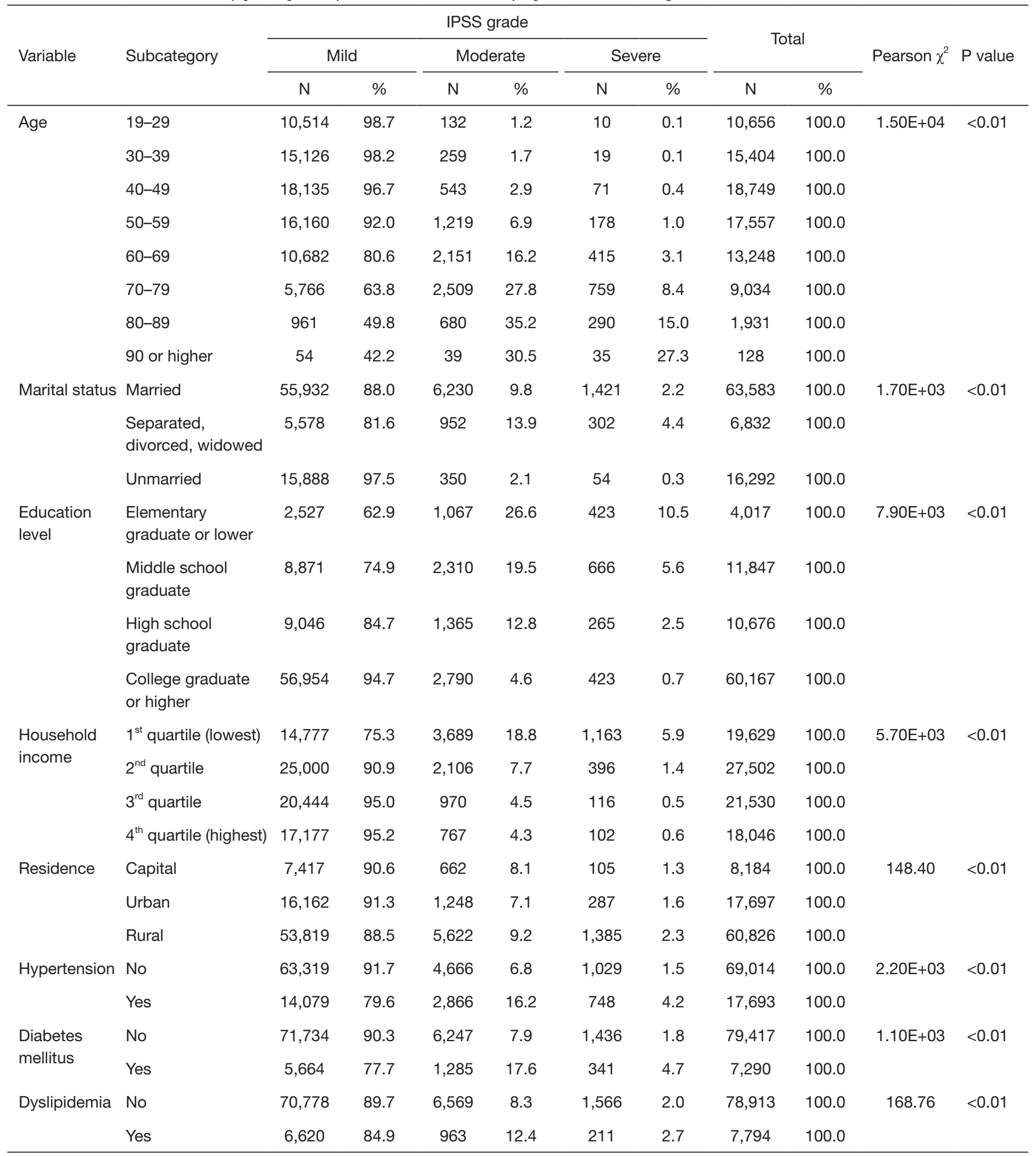

Table 1 (continued) 
Table 1 (continued)

\begin{tabular}{|c|c|c|c|c|c|c|c|c|c|c|c|}
\hline \multirow{3}{*}{ Variable } & \multirow{3}{*}{ Subcategory } & \multicolumn{6}{|c|}{ IPSS grade } & \multirow{2}{*}{\multicolumn{2}{|c|}{ Total }} & \multirow{3}{*}{ Pearson $\chi^{2}$} & \multirow{3}{*}{$P$ value } \\
\hline & & \multicolumn{2}{|c|}{ Mild } & \multicolumn{2}{|c|}{ Moderate } & \multicolumn{2}{|c|}{ Severe } & & & & \\
\hline & & $\mathrm{N}$ & $\%$ & $\mathrm{~N}$ & $\%$ & $\mathrm{~N}$ & $\%$ & $\mathrm{~N}$ & $\%$ & & \\
\hline \multirow{4}{*}{$\begin{array}{l}\text { Alcohol } \\
\text { consumption }\end{array}$} & Non-drinker & 13,683 & 80.2 & 2,588 & 15.2 & 787 & 4.6 & 17,058 & 100.0 & \multirow[t]{4}{*}{$2.70 \mathrm{E}+03$} & \multirow[t]{4}{*}{$<0.01$} \\
\hline & $\begin{array}{l}\text { Less than once per } \\
\text { month }\end{array}$ & 6,540 & 89.3 & 647 & 8.8 & 137 & 1.9 & 7,324 & 100.0 & & \\
\hline & $\begin{array}{l}2 \text { to } 4 \text { times per } \\
\text { month }\end{array}$ & 20,153 & 94.1 & 1,070 & 5.0 & 203 & 0.9 & 21,426 & 100.0 & & \\
\hline & $\begin{array}{l}2 \text { to } 3 \text { times per } \\
\text { week }\end{array}$ & 19,389 & 93.1 & 1,242 & 6.0 & 189 & 0.9 & 20,820 & 100.0 & & \\
\hline \multirow{3}{*}{ Smoking } & Former smoker & 23,016 & 83.8 & 3,567 & 13.0 & 896 & 3.3 & 27,479 & 100.0 & \multirow{3}{*}{$1.30 \mathrm{E}+03$} & \multirow{3}{*}{$<0.01$} \\
\hline & Non-daily smoker & 2,168 & 92.6 & 137 & 5.8 & 37 & 1.6 & 2,342 & 100.0 & & \\
\hline & Daily smoker & 33,820 & 91.9 & 2,434 & 6.6 & 541 & 1.5 & 36,795 & 100.0 & & \\
\hline \multirow[t]{2}{*}{ IPSS voiding } & No & 76,317 & 97.9 & 1,653 & 2.1 & - & 0.0 & 77,970 & 100.0 & \multirow[t]{2}{*}{$6.10 \mathrm{E}+04$} & \multirow[t]{2}{*}{$<0.01$} \\
\hline & Yes & 1,081 & 12.4 & 5,879 & 67.3 & 1,777 & 20.3 & 8,737 & 100.0 & & \\
\hline \multirow[t]{2}{*}{ IPSS storage } & No & 74,574 & 97.4 & 1,976 & 2.6 & 40 & 0.1 & 76,590 & 100.0 & \multirow[t]{2}{*}{$4.60 \mathrm{E}+04$} & \multirow[t]{2}{*}{$<0.01$} \\
\hline & Yes & 2,824 & 27.9 & 5,556 & 54.9 & 1,737 & 17.2 & 10,117 & 100.0 & & \\
\hline
\end{tabular}

significant differences in all independent variables and covariates (all $\mathrm{P}<0.01$ ).

Results of multivariable analysis on IPSS grade, voiding, storage, and nocturia symptoms are shown in Tables 2-5, respectively. Those who smoked in the past but currently quitted and those who were daily smokers showed significantly increased risk of having the worst IPSS grade (past smoker, OR: $1.26,95 \%$ CI: 1.14 to $1.39, \mathrm{P}<0.01$; daily smoker, OR: $1.21,95 \%$ CI: 1.10 to $1.34, \mathrm{P}<0.01$; Table 2 ), having voiding symptoms (past smoker, OR: 1.31, 95\% CI: 1.19 to $1.45, \mathrm{P}<0.01$; daily smoker, OR: $1.31,95 \%$ CI: 1.18 to $1.46, \mathrm{P}<0.01$; Table 3), and having storage symptoms (past smoker, OR: 1.19, 95\% CI: 1.08 to $1.30, \mathrm{P}<0.01$; daily smoker, OR: 1.13 , 95\% CI: 1.03 to 1.24; $\mathrm{P}=0.01$; Table 4) compared to subjects who never smoked. Regarding nocturia symptoms, past smoker showed increased risk of having nocturia symptoms (OR: 1.09, 95\% CI: 1.03 to
$1.15, \mathrm{P}<0.01)$. However, daily smoker showed significantly decreased risk of having nocturia symptoms compared to subjects who were never smokers (OR: 0.79, 95\% CI: 0.75 to $0.84, \mathrm{P}<0.01)($ Table 5$)$.

Those who drank alcohol at least once per month were significantly associated with decreased risk of having the worst IPSS grade (once per month, OR: 0.80, 95\% CI: 0.68 to $0.93, \mathrm{P}<0.01 ; 2$ to 4 times per month, OR: $0.72,95 \% \mathrm{CI}$ : 0.65 to $0.80, \mathrm{P}<0.01 ; 2$ to 3 times per week, OR: $0.80,95 \%$ CI: 0.72 to $0.89, \mathrm{P}<0.01$; 4 or more times per week, OR: $0.90,95 \%$ CI: 0.81 to $0.99, \mathrm{P}=0.03$, Table 2), having voiding symptoms (once per month, OR: $0.82,95 \%$ CI: 0.70 to 0.95 , $\mathrm{P}=0.01 ; 2$ to 4 times per month, OR: $0.80,95 \%$ CI: 0.72 to $0.90, \mathrm{P}<0.01 ; 2$ to 3 times per week, OR: $0.79,95 \% \mathrm{CI}$ : 0.71 to $0.88, \mathrm{P}<0.01 ; 4$ or more times per week, OR: 0.90 , $95 \%$ CI: 0.81 to $0.99, \mathrm{P}=0.03$; Table 3), and having storage symptoms (once per month, OR: $0.80,95 \%$ CI: 0.69 to 0.93 , 
Table 2 Association between alcohol consumption or smoking and IPSS grade

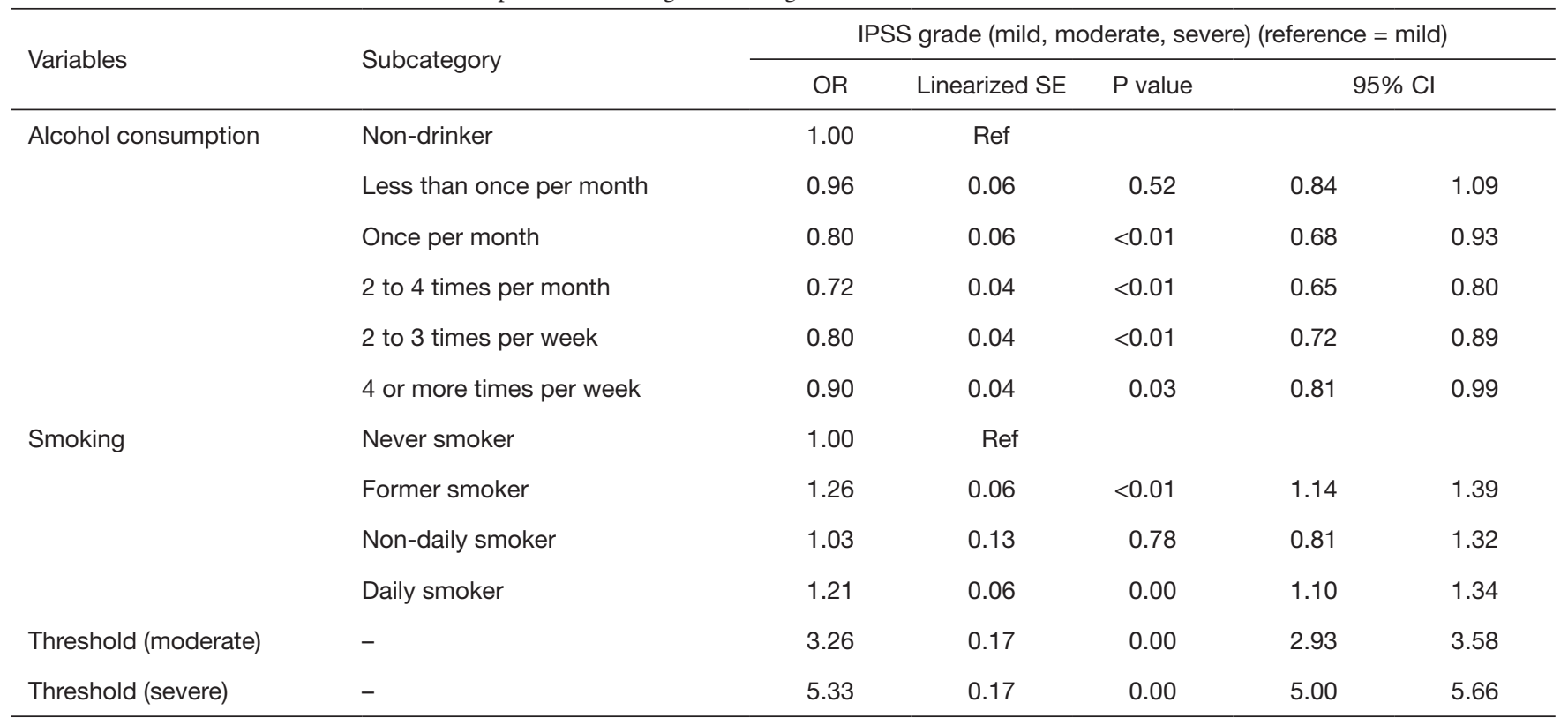

The KCHS as a sample survey was analyzed by study subject and with applied weight calculated in production of the sample design weight and benchmark weight. Strata with single sampling unit centered at overall mean. Sample size =86,707, weighted =16,625,969. Model adjusted for age, marital status, education level, household income, residence, hypertension, diabetes mellitus, and dyslipidemia. KCHS, Korean Community Health Survey; IPSS, International Prostate Symptom Score; OR, odds ratio; CI, confidence interval; SE, standard error; Ref, reference.

Table 3 Association between alcohol consumption or smoking and IPSS voiding

\begin{tabular}{|c|c|c|c|c|c|c|}
\hline Variable & Subcategory & \multicolumn{5}{|c|}{ IPSS voiding (yes $=5$ or higher) $($ reference $=$ no) } \\
\hline \multirow{5}{*}{$\begin{array}{l}\text { Alcohol } \\
\text { consumption }\end{array}$} & Non-drinker & 1.00 & Ref & & & \\
\hline & Less than once per month & 1.00 & 0.07 & 0.95 & 0.88 & 1.14 \\
\hline & Once per month & 0.82 & 0.06 & 0.01 & 0.70 & 0.95 \\
\hline & 2 to 3 times per week & 0.79 & 0.04 & $<0.01$ & 0.71 & 0.88 \\
\hline & 4 or more times per week & 0.90 & 0.05 & 0.03 & 0.81 & 0.99 \\
\hline \multirow[t]{2}{*}{ Smoking } & Never smoker & 1.00 & Ref & & & \\
\hline & Former smoker & 1.31 & 0.07 & $<0.01$ & 1.19 & 1.45 \\
\hline
\end{tabular}

The KCHS as a sample survey was analyzed by study subject and with applied weight calculated in production of the sample design weight and benchmark weight. Strata with single sampling unit centered at overall mean. Sample size =86,707, weighted =16,625,969. Model adjusted for age, marital status, education level, household income, residence, hypertension, diabetes mellitus, and dyslipidemia. KCHS, Korean Community Health Survey; IPSS, International Prostate Symptom Score; OR, odds ratio; CI, confidence interval; SE, standard error; Ref, reference. 
Table 4 Association between alcohol consumption or smoking and IPSS storage

\begin{tabular}{|c|c|c|c|c|c|c|}
\hline Variable & Subcategory & \multicolumn{5}{|c|}{ IPSS Storage (yes =4 or higher) (reference = no) } \\
\hline \multirow{5}{*}{$\begin{array}{l}\text { Alcohol } \\
\text { consumption }\end{array}$} & Non-drinker & 1.00 & Ref & & & \\
\hline & Less than once per month & 0.93 & 0.06 & 0.28 & 0.82 & 1.06 \\
\hline & Once per month & 0.80 & 0.06 & 0.01 & 0.69 & 0.93 \\
\hline & 2 to 3 times per week & 0.81 & 0.04 & $<0.01$ & 0.74 & 0.90 \\
\hline & 4 or more times per week & 0.95 & 0.05 & 0.32 & 0.87 & 1.05 \\
\hline \multirow[t]{2}{*}{ Smoking } & Never smoker & 1.00 & Ref & & & \\
\hline & Former smoker & 1.19 & 0.06 & $<0.01$ & 1.08 & 1.30 \\
\hline
\end{tabular}

The $\mathrm{KCHS}$ as a sample survey was analyzed by study subject and with applied weight calculated in production of the sample design weight and benchmark weight. Strata with single sampling unit centered at overall mean. Sample size $=86,707$, weighted $=16,625,969$. Model adjusted for age, marital status, education level, household income, residence, hypertension, diabetes mellitus, and dyslipidemia. KCHS, Korean Community Health Survey; IPSS, International Prostate Symptom Score; OR, odds ratio; Cl, confidence interval; SE, standard error; Ref, reference.

Table 5 Association between alcohol consumption or smoking and nocturia

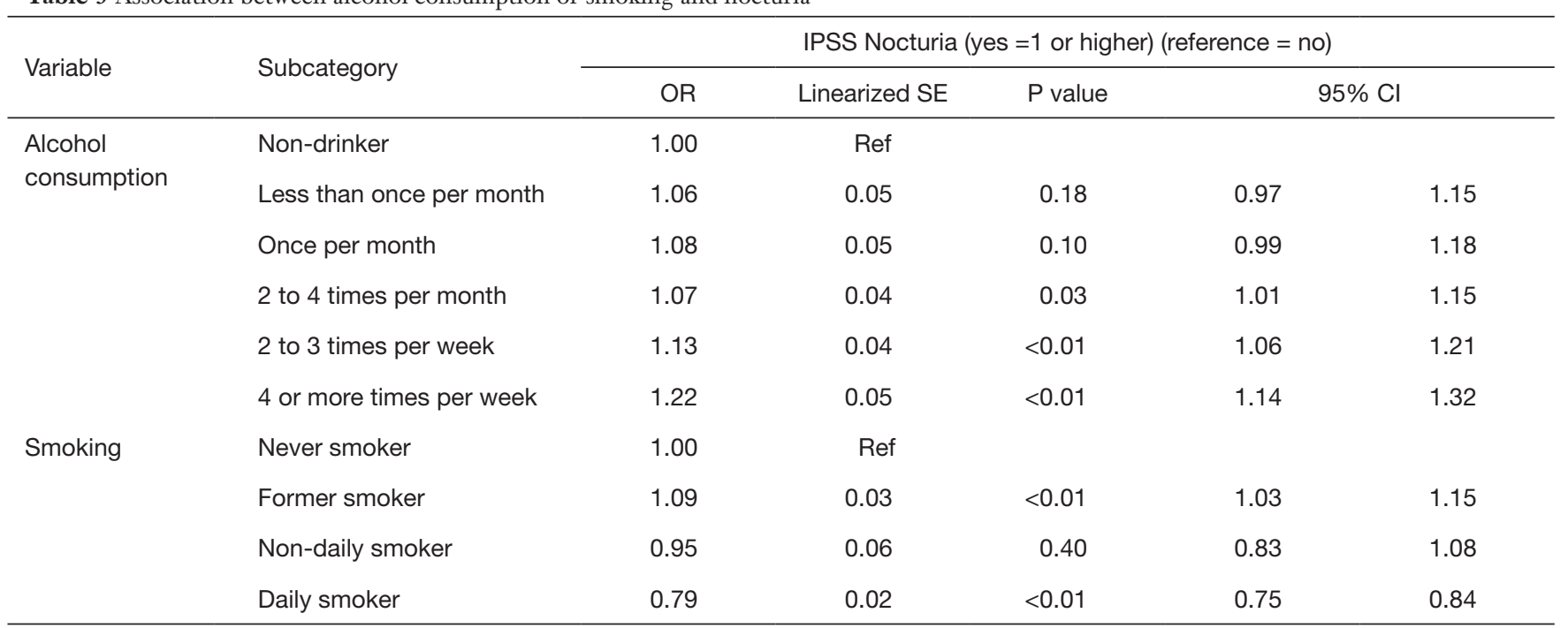

The KCHS as a sample survey was analyzed by study subject and with applied weight calculated in production of the sample design weight and benchmark weight. Strata with single sampling unit centered at overall mean. Sample size =86,707, weighted =16,625,969. Model adjusted for age, marital status, education level, household income, residence, hypertension, diabetes mellitus, and dyslipidemia. KCHS, Korean Community Health Survey; IPSS, International Prostate Symptom Score; OR; odds ratio; CI, confidence interval; SE, standard error; Ref, reference. 
$\mathrm{P}=0.01 ; 2$ to 4 times per month, OR: $0.77,95 \% \mathrm{CI}: 0.69$ to $0.85, \mathrm{P}<0.01 ; 2$ to 3 times per week, OR: $0.81,95 \% \mathrm{CI}: 0.74$ to $0.90, \mathrm{P}<0.01$; Table 4 ) compared to subjects in the nondrinker group. On the other hand, those who drank alcohol at least twice per month were significantly associated with increased risk of having nocturia symptoms compared to subjects in the non-drinker group ( 2 to 4 times per month, OR: $1.07,95 \%$ CI: 1.01 to $1.15, \mathrm{P}=0.03 ; 2$ to 3 times per week, OR: 1.13 , 95\% CI: 1.06 to $1.21, \mathrm{P}<0.01$; 4 or more times per week, OR: 1.22 , 95\% CI: 1.14 to 1.32 ; Table 5).

\section{Discussion}

The main focus of this study was to investigate relationships of LUTS with cigarette smoking and alcohol intake using large national representative population data so that more firmed evidence about their relationship could be obtained. Considering conflicting findings about their relationships, this study could provide supportive information by overcoming low population-based sample issue of previous studies. Our results suggest a positive role of alcohol drinking and a negative role of cigarette smoking on diverse LUTS categories including general LUTS (IPSS severity), storage LUTS, and voiding LUTS. The most interesting finding of this study is that cigarette smoking and alcohol intake could affect nocturia. This is a novel finding. Only limited literature has scope of this finding.

Although one recent systematic review concluded that modest alcohol intake was a positive factor for male LUTS as it decreased the risk of LUTS compared to non-drinking as moderate alcohol intake was associated with decreased risk of BPH diagnosis and undergoing surgery (2), two large longitudinal prospective studies showed opposite findings $(6,7)$. One study showed that daily alcohol drinking had no effect on LUTS severity in their 4-year follow-up study (6) while another study showed a positive effect of alcohol drinking on LUTS severity in their 7-year follow-up study (7). However, heavy alcohol intake had negative effect on LUTS including incontinence, voiding, and storage LUTS (4). This J-shaped association between alcohol intake and LUTS severity was also found in a large population study (8). It reported that the risk for moderate and severe LUTS was the lowest in modest drinkers but the highest in heavy drinkers compared to non-drinkers (8).

Our study showed a positive effect of alcohol intake on LUTS severity by IPSS grade. Not only moderate drinkers, but also heavy drinkers (alcohol consumption: 4 or more times per week) showed significantly reduced risk for LUTS severity. Alcohol intake also had positive effects on voiding LUTS and storage LUTS. Among LUTS, association between alcohol intake and $\mathrm{OAB}$ showed more inconsistent results that that between alcohol intake and general LUTS. In two large population studies, one study showed negative role of alcohol intake in OAB (9) while the other study did not show any association (10). In our study, alcohol intake showed a positive role in storage LUTS by IPSS, even heavy drinkers showed decreasing OR for storage LUTS, although the decrease was not statistically significant.

There are only a few studies about the relationship between alcohol intake and nocturia. One cohort study showed no association between alcohol intake and nocturia (9). However, another study showed that modest alcohol intake was associated with lower risk of nocturia (11). In our study, alcohol intake showed negative effect of any level of drinking on nocturia. All results were statistically significant findings compared to non-alcohol drinkers. Our study is the first one to show a negative effect of alcohol intake on nocturia.

Possible mechanisms for the relationship between alcohol intake and LUTS include increased sympathetic tone activity, diuretic effect, and changed androgen levels $(12,13)$. Positive effect of alcohol intake on LUTS could be explained by diuretic effect and low androgen level while negative effect could be explained by diuretic effect and increased sympathetic tone activity. Diuretic effect could serve as both positive and negative factor in the effect of alcohol intake on LUTS. Individual differences in genetic polymorphism of the aldehyde dehydrogenase 2 (ALDH2) gene might also have associations with $\mathrm{BPH}$ (14).

The relationship between cigarette smoking and LUTS has more limited evidence than that between alcohol and LUTS. Several studies have shown a negative effect of cigarette smoking on LUTS (2), although other studies have shown no association between the degree of smoking and LUTS $(12,15)$. In our study, cigarette smoking showed negative effect on all LUTS except nocturia. The degree of smoking was also related to the severity of LUTS in that it had negative effects on all LUTS except nocturia.

The possible mechanism for the effect of cigarette smoking on LUTS has been investigated in many experimental studies. These studies have suggested that cigarette smoking could increase androgen level $(16,17)$, increase the sympathetic tone activity by nicotine receptor $(18,19)$, and cause imbalance in hormone and nutrient between muscle and collagen (20). Other studies support that the negative effect of cigarette smoking on nocturia is especially related to metabolic syndrome (21). However, 
Bing et al. (22) in their Danish population study reported that smoking was inversely associated with nocturia in both crude and adjusted models. Although significance disappeared compared to severe nocturia of $\geq 2$ or $\geq 3$ voids per night, the significance appeared in the ordinal logistic model. Seim et al. (23) have also reported an inverse association between smoking and nocturia, supporting a positive or protective effect of smoking on nocturia. Although we could not adjust metabolic syndrome factor, smoking was inversely associated with nocturia after adjustment of possible covariates including body mass index (BMI). This protective effect of smoking could be explained by increased arginine vasopressin due to increased nicotine, thus decreasing nocturnal urine production $(24,25)$.

Although we have performed a thorough analysis, this study still has several limitations. First, cross-sectional study design hampered the establishment of a causal effect of alcohol intake or cigarette smoking on the severity of LUTS. Second, subjective questionnaire was used to access the degree of alcohol intake and cigarette smoking. However, estimating the degree of drinking and smoking without questionnaire is difficult. Lastly, these data did not include biological data including prostate size or chemistry profiles. They did not include objective measurement of LUTS such as uroflowmetry or frequency volume chart either.

\section{Conclusions}

This large population-based study suggests updated evidence about relationships of LUTS with alcohol intake and cigarette smoking. Alcohol showed positive effect on LUTS except nocturia whereas cigarette smoking had negative effect on LUTS except nocturia. Alcohol increased the risk of nocturia while smoking decreased the risk of nocturia. Further evidence using prospective trial is needed to support our study findings.

\section{Acknowledgments}

Funding: This work was supported by Soonchunhyang University Research Fund.

\section{Footnote}

Conflicts of Interest: All authors have completed the ICMJE uniform disclosure form (available at http://dx.doi. org/10.21037/tau.2020.03.07). The authors have no conflicts of interest to declare.
Ethical Statement: The authors are accountable for all aspects of the work in ensuring that questions related to the accuracy or integrity of any part of the work are appropriately investigated and resolved. Procedures of this study were reviewed and approved by the Institutional Review Board of Soonchunhyang University Seoul Hospital with a waiver for informed consent (2018-12-011). KCHS data are openly accessible at national public repository (http://chs.cdc.go.kr). There were no confidentiality risks to participants of this study because all KCHS data were deidentified.

Open Access Statement: This is an Open Access article distributed in accordance with the Creative Commons Attribution-NonCommercial-NoDerivs 4.0 International License (CC BY-NC-ND 4.0), which permits the noncommercial replication and distribution of the article with the strict proviso that no changes or edits are made and the original work is properly cited (including links to both the formal publication through the relevant DOI and the license). See: https://creativecommons.org/licenses/by-nc$\mathrm{nd} / 4.0 \%$.

\section{References}

1. Gormley EA, Lightner DJ, Faraday M, et al. Diagnosis and treatment of overactive bladder (non-neurogenic) in adults: AUA/SUFU guideline amendment. J Urol 2015;193:1572-80.

2. Bradley CS, Erickson BA, Messersmith EE, et al. Evidence of the Impact of Diet, Fluid Intake, Caffeine, Alcohol and Tobacco on Lower Urinary Tract Symptoms: A Systematic Review. J Urol 2017;198:1010-20.

3. Maserejian NN, Kupelian V, Miyasato G, et al. Are physical activity, smoking and alcohol consumption associated with lower urinary tract symptoms in men or women? Results from a population based observational study. J Urol 2012;188:490-5.

4. Joseph MA, Harlow SD, Wei JT, et al. Risk factors for lower urinary tract symptoms in a population-based sample of African-American men. Am J Epidemiol 2003;157:906-14.

5. Calogero AE, Burgio G, Condorelli RA, et al. Epidemiology and risk factors of lower urinary tract symptoms/benign prostatic hyperplasia and erectile dysfunction. Aging Male 2019;22:12-9.

6. Wong SY, Woo J, Leung JC, et al. Depressive symptoms and lifestyle factors as risk factors of lower urinary tract 
symptoms in Southern Chinese men: a prospective study. Aging Male 2010;13:113-9.

7. Kristal AR, Arnold KB, Schenk JM, et al. Dietary patterns, supplement use, and the risk of symptomatic benign prostatic hyperplasia: results from the prostate cancer prevention trial. Am J Epidemiol 2008;167:925-34.

8. Oh MJ, Eom CS, Lee HJ, et al. Alcohol consumption shows a J-shaped association with lower urinary tract symptoms in the general screening population. J Urol 2012;187:1312-7.

9. Schneider T, Marschall-Kehrel D, Hanisch JU, et al. Do gender, age or lifestyle factors affect responses to antimuscarinic treatment in overactive bladder patients? Int J Clin Pract 2010;64:1287-93.

10. Maserejian NN, Giovannucci EL, McVary KT, et al. Dietary macronutrient and energy intake and urinary incontinence in women. Am J Epidemiol 2010;171:1116-25.

11. Shiri R, Hakama M, Hakkinen J, et al. The effects of lifestyle factors on the incidence of nocturia. J Urol 2008;180:2059-62.

12. Platz EA, Rimm EB, Kawachi I, et al. Alcohol consumption, cigarette smoking, and risk of benign prostatic hyperplasia. Am J Epidemiol 1999;149:106-15.

13. van de Borne P, Mark AL, Montano N, et al. Effects of alcohol on sympathetic activity, hemodynamics, and chemoreflex sensitivity. Hypertension 1997;29:1278-83.

14. Jang HS, Kim JS, Kim SS, et al. Relationship Between Alcohol Consumption and Prostatic Hyperplasia According to Facial Flushing After Drinking in Korean Men. Korean J Fam Med 2017;38:93-8.

15. Goh HJ, Kim SA, Nam JW, et al. Community-based research on the benign prostatic hyperplasia prevalence rate in Korean rural area. Korean J Urol 2015;56:68-75.

16. Celermajer DS, Sorensen KE, Georgakopoulos D, et al. Cigarette smoking is associated with dose-related and potentially reversible impairment of endotheliumdependent dilation in healthy young adults. Circulation

Cite this article as: Noh JW, Yoo KB, Kim KB, Kim JH, Kwon YD. Association between lower urinary tract symptoms and cigarette smoking or alcohol drinking. Transl Androl Urol 2020;9(2):312-321. doi: 10.21037/tau.2020.03.07
1993;88:2149-55.

17. Huang YC, Chin CC, Chen CS, et al. Chronic Cigarette Smoking Impairs Erectile Function through Increased Oxidative Stress and Apoptosis, Decreased nNOS, Endothelial and Smooth Muscle Contents in a Rat Model. PLoS One 2015;10:e140728.

18. Narkiewicz K, van de Borne PJ, Hausberg M, et al. Cigarette smoking increases sympathetic outflow in humans. Circulation 1998;98:528-34.

19. Rohrmann S, Crespo CJ, Weber JR, et al. Association of cigarette smoking, alcohol consumption and physical activity with lower urinary tract symptoms in older American men: findings from the third National Health And Nutrition Examination Survey. BJU Int 2005;96:77-82.

20. Knuutinen A, Kokkonen N, Risteli J, et al. Smoking affects collagen synthesis and extracellular matrix turnover in human skin. Br J Dermatol 2002;146:588-94.

21. De Nunzio C, Brassetti A, Proietti F, et al. Metabolic syndrome and smoking are associated with an increased risk of nocturia in male patients with benign prostatic enlargement. Prostate Cancer Prostatic Dis 2018;21:287-92.

22. Bing MH, Moller LA, Jennum $P$, et al. Nocturia and associated morbidity in a Danish population of men and women aged $60-80$ years. BJU Int 2008;102:808-14; discussion 814-5.

23. Seim A, Hoyo C, Ostbye T, et al. The prevalence and correlates of urinary tract symptoms in Norwegian men: the HUNT study. BJU Int 2005;96:88-92.

24. Fuxe K, Andersson K, Eneroth P, et al. Neuroendocrine actions of nicotine and of exposure to cigarette smoke: medical implications. Psychoneuroendocrinology 1989;14:19-41.

25. Hunsballe JM, Rittig S, Pedersen EB, et al. Smokeless nicotinergic stimulation of vasopressin secretion in patients with persisting nocturnal enuresis and controls. Scand J Urol Nephrol 2001;35:117-21. 